\title{
GLOBAL WELL-POSEDNESS OF THE HALF SPACE PROBLEM OF THE NAVIER-STOKES EQUATIONS IN CRITICAL FUNCTION SPACES OF LIMITING CASE
}

\author{
TONGKEUN CHANG AND BUM JA JIN
}

\begin{abstract}
In this paper, we study the initial-boundary value problem of the Navier-Stokes equations in half-space. Let a solenoidal initial velocity be given in the function space $\dot{B}_{p \infty, 0}^{-1+n / p}\left(\mathbb{R}_{+}^{n}\right)$ for $\frac{n}{3}<p<n$. We prove the global in time existence of weak solution $u \in L^{\infty}\left(0, \infty ; \dot{B}_{p \infty}^{-1+n / p}\left(\mathbb{R}_{+}^{n}\right)\right)$, when the given initial velocity has small norm in function space $\dot{B}_{p \infty, 0}^{-1+n / p}\left(\mathbb{R}_{+}^{n}\right)$, where $\frac{n}{3}<p<n$.
\end{abstract}

2000 Mathematics Subject Classification: primary 35K61, secondary 76D07.

Keywords and phrases: Stokes equations, Navier-Stokes equations, Homogeneous initial boundary value problem, Half-space.

\section{Introduction}

In this paper, we study the initial-boundary value problem of Navier-Stokes equations

$$
\begin{aligned}
& u_{t}-\Delta u+\nabla p=-\operatorname{div}(u \otimes u), \quad \operatorname{div} u=0 \text { in } \mathbb{R}_{+}^{n} \times(0, \infty), \\
& u(x, 0)=u_{0}(x) \quad x \in \mathbb{R}_{+}^{n}, \quad u\left(x^{\prime}, 0, t\right)=0 \quad x^{\prime} \in \mathbb{R}^{n-1}, t \in(0, \infty),
\end{aligned}
$$

where $u=\left(u_{1}, \cdots, u_{n}\right)$ and $p$ are the unknown velocity and pressure, respectively, and $u_{0}=$ $\left(u_{01}, \cdots, u_{0 n}\right)$ is the given initial data.

Because the Navier-Stokes equations are invariant under the scaling

$$
u_{\lambda}(x, t)=\lambda u\left(\lambda x, \lambda^{2} t\right), \quad p_{\lambda}(x, t)=\lambda^{2} p\left(\lambda x, \lambda^{2} t\right), \quad u_{0 \lambda}(x)=\lambda u_{0}(\lambda x),
$$

it is important to study (1.1) in the so-called critical spaces, i.e., the function spaces with norms invariant under the scaling $u(x, t) \rightarrow \lambda u\left(\lambda x, \lambda^{2} t\right)$. The homogeneous Besov space $\dot{B}_{p q}^{-1+\frac{n}{p}}\left(\mathbb{R}_{+}^{n}\right)$ is one of critical spaces.

In this paper, we prove the existence of global time mild solution $u \in L^{\infty}\left(0, \infty ; \dot{B}_{p \infty}^{-1+\frac{n}{p}}\left(\mathbb{R}_{+}^{n}\right)\right)$ of (1.1) for the initial data $u_{0} \in \dot{B}_{p \infty, 0}^{-1+\frac{n}{p}}\left(\mathbb{R}_{+}^{n}\right), \frac{n}{3}<p<n$. See Section 2 for definitions of function spaces.

There are a number of papers dealing with global well-posedness for (1.1) in homogeneous Besov space $\dot{B}_{p q}^{-1+\frac{n}{p}}\left(\mathbb{R}_{+}^{n}\right), 1<p, q<\infty$ (see [6, 7, 8, 9, 13, 14, 16, 23] and the references therein).

The limiting case $1<p<\infty, q=\infty$ has been studied by M. Cannone, F. Planchon, and M. Schonbek [4] for $u_{0} \in L^{3}\left(\mathbb{R}_{+}^{3}\right)$, by H. Amann [2] for $u_{0} \in b_{p, \infty}^{-1+\frac{n}{p}}(\Omega), p>\frac{n}{3}, p \neq n$, where $\Omega$ is

Tongkeun Chang was supported by NRF-2017R1D1A1B03033427 and Bum Ja Jin was supported by NRF2016R1D1A1B03934133. 
a standard domain like $\mathbb{R}^{3}, \mathbb{R}_{+}^{3}$, exterior or bounded domain in $\mathbb{R}^{3}$ and by M. Ri, P. Zhang and Z. Zhang [26] for $u_{0} \in b_{n \infty}^{0}(\Omega)$, where $\Omega$ is $\mathbb{R}^{n}, \mathbb{R}_{+}^{n}$ or bounded domain with smooth boundary, and $b_{p \infty}^{s}(\Omega)$ denotes the completion of the generalized Sobolev space $H_{p}^{s}(\Omega)$ in $B_{p \infty}^{s}(\Omega)$. In particular, in [4], the solution $u \in L_{\frac{1}{2}-\frac{3}{2 p}}^{\infty}\left(0, \infty, L^{3}\left(\mathbb{R}_{+}^{3}\right)\right)$ exists globally in time when $\left\|u_{0}\right\|_{\dot{B}_{p \infty}^{-1+\frac{3}{p}}\left(\mathbb{R}_{+}^{3}\right)}, p>3$ $\left(L^{3}\left(\mathbb{R}_{+}^{n}\right) \subset \dot{B}_{p \infty}^{-1+\frac{3}{p}}\left(\mathbb{R}_{+}^{3}\right)\right)$ is small enough. Recently, H. Kozono and S. Shimizu [22] showed the existence of solution $u \in L^{\infty}\left(0, \infty ; \dot{B}_{p \infty}^{-1+\frac{n}{p}}\left(\mathbb{R}^{n}\right)\right), p>n$ of 1.1 with sufficiently small norm of $u_{0}$ in $\dot{B}_{p \infty, 0}^{-1+\frac{n}{p}}\left(\mathbb{R}^{n}\right)$. See also [1, 15, 18, 21, 24, 25, 28] and the references therein for the initial value problem of Navier-Stokes equations in the half space.

Our study of this paper is motivated by the results in [2, 4, 22, 26]. The following text states our main results.

Theorem 1.1. Let $\frac{n}{3}<p<n$. Assume that $u_{0} \in \dot{B}_{p \infty, 0}^{-1+\frac{n}{p}}\left(\mathbb{R}_{+}^{n}\right)$ with $\operatorname{div} u_{0}=0$. Then, there is $\epsilon_{*}>0$ so that if $\left\|u_{0}\right\|_{\dot{B}_{p_{0} \infty, 0}^{-1+\frac{n}{p_{0}}\left(\mathbb{R}_{+}^{n}\right)}}<\epsilon_{*}$ for some $n<p_{0}<\infty$, then (1.1) has a solution $u \in L^{\infty}\left(0, \infty ; \dot{B}_{p \infty}^{-1+\frac{n}{p}}\left(\mathbb{R}_{+}^{n}\right)\right) \cap L_{\frac{1}{2}-\frac{n}{2 p_{0}}}^{\infty}\left(0, \infty ; L^{p_{0}}\left(\mathbb{R}_{+}^{n}\right)\right)$.

Remark 1.2. In Theorem 1.1 observe that $\dot{B}_{p \infty, 0}^{-1+\frac{n}{p}}\left(\mathbb{R}_{+}^{n}\right) \subset \dot{B}_{p_{0} \infty, 0}^{-1+\frac{n}{p_{0}}}\left(\mathbb{R}_{+}^{n}\right)$ holds (see Theorem 6.5.1 in [3]).

For the proof of Theorem 1.1, it is necessary to study the following initial value problem of the Stokes equations in $\mathbb{R}_{+}^{n} \times(0, \infty)$ :

$$
\begin{array}{r}
u_{t}-\Delta u+\nabla p=f, \quad \operatorname{div} u=0 \text { in } \mathbb{R}_{+}^{n} \times(0, \infty), \\
\left.u\right|_{t=0}=u_{0},\left.\quad u\right|_{x_{n}=0}=0,
\end{array}
$$

where $f=\operatorname{div} \mathcal{F}$.

In [17], M. Giga, Y. Giga and H. Sohr showed that if $f \in L^{q}\left(0, T ; \hat{D}\left(A_{p}^{-\alpha}\right)\right)$ and $u_{0}=0$ then the solution $u$ of Stokes equations (1.2) satisfies that for $0<\alpha<1$,

$$
\int_{0}^{T}\left(\left\|\left(\frac{d}{d t}\right)^{1-\alpha} u(t)\right\|_{L^{p}(\Omega)}^{q}+\left\|A_{p}^{1-\alpha} u(t)\right\|_{L^{p}(\Omega)}^{q}\right) d t \leq c(p, q, \Omega, \alpha) \int_{0}^{T}\left\|A_{p}^{-\alpha} f(t)\right\|_{L^{p}(\Omega)}^{q} d t,
$$

where $A_{p}$ is Stokes operator in $\Omega$ for standard domain $\Omega$ such as bounded domain, exterior domain or half space, and $\hat{D}(T)$ is the completion of $D(T)$ in the homogeneous norm $\|T \cdot\|$. In particular, if $f=\operatorname{div} \mathcal{F}$ with $\mathcal{F} \in L^{q}\left(0, T ; L_{\sigma}^{p}(\Omega)\right)$ then

$$
\int_{0}^{T}\left(\left\|\left(\frac{d}{d t}\right)^{\frac{1}{2}} u(t)\right\|_{L^{p}(\Omega)}^{q}+\|\nabla u(t)\|_{L^{p}(\Omega)}^{q}\right) d t \leq c(p, q, \Omega) \int_{0}^{T}\|\mathcal{F}(t)\|_{L^{p}(\Omega)}^{q} d t .
$$

H. Koch and V. A. Solonnikov [19] showed the unique local in time existence of solution $u \in$ $L^{q}\left(0, T ; L^{q}\left(\mathbb{R}_{+}^{3}\right)\right)$ of (1.2) when $f=\operatorname{div} \mathcal{F}, \mathcal{F} \in L^{q}\left(0, T ; L^{q}\left(\mathbb{R}_{+}^{3}\right)\right)$ and $u_{0}=0$. See also [18, 20, 21, 28, 29] and the references therein. 
The following theorem states our result on the unique solvability of the Stokes equations (1.2).

Theorem 1.3. Let $1<p_{1} \leq p<\infty$ and $\alpha>0$. Let $u_{0} \in \dot{B}_{p \infty, 0}^{-\alpha}\left(\mathbb{R}_{+}^{n}\right)$ with $\operatorname{div} u_{0}=0$ and $f=\operatorname{div} \mathcal{F}$.

(1) Let $-1+\alpha<\frac{n}{p_{1}}-\frac{n}{p}<1$ and $\mathcal{F} \in L_{\frac{1}{2} \alpha+\frac{1}{2}-\frac{n}{2}\left(\frac{1}{p_{1}}-\frac{1}{p}\right)}^{\infty}\left(0, \infty, L^{p_{1}}\left(\mathbb{R}_{+}^{n}\right)\right)$. There is a solution u of (1.2) with

$$
\|u\|_{L_{\frac{1}{2} \alpha}^{\infty}\left(0, \infty ; L^{p}\left(\mathbb{R}_{+}^{n}\right)\right)} \leq c\left(\left\|u_{0}\right\|_{\dot{B}_{p \infty, 0}^{-\alpha}\left(\mathbb{R}_{+}^{n}\right)}+\|\mathcal{F}\|_{L_{\frac{1}{2} \alpha+\frac{1}{2}-\frac{n}{2}\left(\frac{1}{p_{1}}-\frac{1}{p}\right)}^{\infty}\left(0, \infty ; L^{\left.p_{1}\left(\mathbb{R}_{+}^{n}\right)\right)}\right) .} .\right.
$$

(2) Let $0<\alpha<2$ such that $1+\frac{n-1}{p}<\frac{n}{p_{1}}<1+\frac{n}{p}$ and $\mathcal{F} \in L_{\frac{1}{2}-\frac{n}{2 p_{1}}+\frac{n}{2 p}}^{\infty}\left(0, \infty ; \dot{B}_{p_{1} \infty}^{\alpha}\left(\mathbb{R}_{+}^{n}\right)\right)$. There is a solution $u$ of (1.2) with

$$
\|u\|_{L^{\infty}\left(0, \infty ; \dot{B}_{p \infty}^{\alpha}\left(\mathbb{R}_{+}^{n}\right)\right)} \leq c\left(\left\|u_{0}\right\|_{\dot{B}_{p \infty, 0}^{\alpha}\left(\mathbb{R}_{+}^{n}\right)}+\|\mathcal{F}\|_{L_{\frac{1}{2}-\frac{n}{2 p_{1}}+\frac{n}{2 p}}\left(0, \infty ; \dot{B}_{p_{1} \infty}^{\alpha}\left(\mathbb{R}_{+}^{n}\right)\right)}\right) .
$$

This paper is organized as follows. In Section 2 , we introduce the function spaces and definition of the weak solutions of Stokes equations and Navier-Stokes equations. In Section 3, various estimates of operators related to a Newtonian kernel and Gaussian kernel are given. In Section 4, we complete the proof of Theorem 1.3. In Section 5, we give the proof of Theorem 1.1 while applying the estimates in Theorem 1.3 to the approximate solutions.

\section{Notations, Function SPACES AND DEFinitions of WEAK SOLUtions}

We denote by $x^{\prime}$ and $x=\left(x^{\prime}, x_{n}\right)$ the points of the spaces $\mathbb{R}^{n-1}$ and $\mathbb{R}^{n}$, respectively. The multiple derivatives are denoted by $D_{x}^{k} D_{t}^{m}=\frac{\partial^{|k|}}{\partial x^{k}} \frac{\partial^{m}}{\partial t}$ for multi-index $k$ and nonnegative integers $m$. Throughout this paper we denote by $c$ various generic constants.

For $s \in \mathbb{R}$ and $1 \leq p, q \leq \infty$, we denote $\dot{H}_{p}^{s}\left(\mathbb{R}^{n}\right)$ and $\dot{B}_{p q}^{s}\left(\mathbb{R}^{n}\right)$ the generalized homogeneous Sobolev spaces(space of Bessel potentials) and the homogeneous Besov spaces in $\mathbb{R}^{n}$, respectively (see [3, 31] for the definition of function spaces). Denoted by $\dot{H}_{p}^{s}\left(\mathbb{R}_{+}^{n}\right)$ and $\dot{B}_{p q}^{s}\left(\mathbb{R}_{+}^{n}\right)$ are the restrictions of $\dot{H}_{p}^{s}\left(\mathbb{R}^{n}\right)$ and $\dot{B}_{p q}^{s}\left(\mathbb{R}^{n}\right)$, respectively, with norms

$$
\begin{gathered}
\|f\|_{\dot{H}_{p}^{s}\left(\mathbb{R}_{+}^{n}\right)}=\inf \left\{\|F\|_{\dot{H}_{p}^{s}\left(\mathbb{R}^{n}\right)}|F|_{\mathbb{R}_{+}^{n}}=f, F \in \dot{H}_{p}^{s}\left(\mathbb{R}^{n}\right)\right\}, \\
\|f\|_{\dot{B}_{p q}^{s}\left(\mathbb{R}_{+}^{n}\right)}=\inf \left\{\|F\|_{\dot{B}_{p q}^{s}\left(\mathbb{R}^{n}\right)}|F|_{\mathbb{R}_{+}^{n}}=f, F \in \dot{B}_{p q}^{s}\left(\mathbb{R}^{n}\right)\right\} .
\end{gathered}
$$

For a non-negative integer $k, \dot{H}_{p}^{k}\left(\mathbb{R}_{+}^{n}\right)=\left\{f \mid \sum_{|l|=k}\left\|D^{l} f\right\|_{L^{p}\left(\mathbb{R}_{+}^{n}\right)}<\infty\right\}$. In particular, $\dot{H}_{p}^{0}\left(\mathbb{R}_{+}^{n}\right)=$ $L^{p}\left(\mathbb{R}_{+}^{n}\right)$.

For $s \in \mathbb{R}$, we denote $\dot{B}_{p q, 0}^{s}\left(\mathbb{R}_{+}^{n}\right), 1 \leq p, q \leq \infty$ by

$$
\dot{B}_{p q, 0}^{s}\left(\mathbb{R}_{+}^{n}\right)=\left\{f \in \dot{B}_{p q}^{s}\left(\mathbb{R}_{+}^{n}\right) \mid \tilde{f} \in \dot{B}_{p q}^{s}\left(\mathbb{R}^{n}\right)\right\},
$$

where $\tilde{f}$ is the zero extension of $f$ over $\mathbb{R}^{n}$. Note that $\|\tilde{f}\|_{\dot{B}_{p q}^{s}\left(\mathbb{R}^{n}\right)} \leq c\|f\|_{\dot{B}_{p q, 0}^{s}\left(\mathbb{R}_{+}^{n}\right)}$. 
Note that for $s \geq 0, \dot{B}_{p q, 0}^{-s}\left(\mathbb{R}_{+}^{n}\right), 1<p, q \leq \infty$ is the dual space of $\dot{B}_{p^{\prime} q^{\prime}}^{s}\left(\mathbb{R}_{+}^{n}\right)$, that is, $\dot{B}_{p q, 0}^{-s}\left(\mathbb{R}_{+}^{n}\right)=\left(\dot{B}_{p^{\prime} q^{\prime}}^{s}\left(\mathbb{R}_{+}^{n}\right)\right)^{*}$, where $\frac{1}{p}+\frac{1}{p^{\prime}}=1$ and $\frac{1}{q}+\frac{1}{q^{\prime}}=1$.

For the Banach space $X$, we denote by $L_{\beta}^{\infty}(0, \infty ; X), \beta \geq 0$ the usual Bochner space with norm

$$
\|f\|_{L_{\beta}^{\infty}(0, \infty ; X)}:=\sup _{0<t<\infty} t^{\beta}\|f(t)\|_{X} .
$$

For $1 \leq q \leq \infty$ and $0<\theta<1$, we denote by $(X, Y)_{\theta, q}$ and $[X, Y]_{\theta}$ the real interpolation and complex interpolation of the Banach spaces $X$ and $Y$. In particular, for $0<\theta<1, \alpha, \alpha_{1}, \alpha_{2} \in \mathbb{R}$ and $1 \leq p, q, r \leq \infty$,

$$
\begin{aligned}
{\left[\dot{H}_{p}^{\alpha_{1}}\left(\mathbb{R}_{+}^{n}\right), \dot{H}_{p}^{\alpha_{2}}\left(\mathbb{R}_{+}^{n}\right)\right]_{\theta} } & =\dot{H}_{p}^{\alpha}\left(\mathbb{R}_{+}^{n}\right), \\
{\left[\dot{B}_{p_{1}}^{\alpha}\left(\mathbb{R}_{+}^{n}\right), \dot{B}_{p q}^{\alpha_{2}}\left(\mathbb{R}_{+}^{n}\right)\right]_{\theta} } & =\dot{B}_{p r}^{\alpha}\left(\mathbb{R}_{+}^{n}\right), \\
\left(\dot{H}_{p}^{\alpha_{1}}\left(\mathbb{R}_{+}^{n}\right), \dot{H}_{p}^{\alpha_{2}}\left(\mathbb{R}_{+}^{n}\right)\right)_{\theta, r} & =\dot{B}_{p r}^{\alpha}\left(\mathbb{R}_{+}^{n}\right),
\end{aligned}
$$

when $\alpha=\theta \alpha_{1}+(1-\theta) \alpha_{2}$. See Theorem 6.4.5, Theorem 5.1.2 and Theorem 5.6.2 in [3].

From now, we denote $\dot{B}_{p \infty}^{\beta}:=\dot{B}_{p \infty}^{\beta}\left(\mathbb{R}_{+}^{n}\right), \dot{H}_{p}^{\beta}:=\dot{H}_{p}^{\beta}\left(\mathbb{R}_{+}^{n}\right), L_{\alpha}^{\infty} B_{p \infty}^{\beta}:=L_{\alpha}^{\infty}\left(0, \infty ; \dot{B}_{p \infty}^{\beta}\left(\mathbb{R}_{+}^{n}\right)\right)$ and $L_{\alpha}^{\infty} L^{p}:=L_{\alpha}^{\infty}\left(0, \infty ; \dot{L}^{p}\left(\mathbb{R}_{+}^{n}\right)\right)$.

\section{Preliminary Estimates.}

3.1. Newtonian potential. The fundamental solution of the Laplace equation in $\mathbb{R}^{n}$ is denoted by

$$
N(x)= \begin{cases}\frac{1}{\omega_{n}(2-n)|x|^{n-2}} & \text { if } n \geq 3, \\ \frac{1}{2 \pi} \ln |x| & \text { if } n=2,\end{cases}
$$

$\omega_{n}$ is the surface area of the unit sphere in $\mathbb{R}^{n}$.

Lemma 3.1. For $\alpha \geq 0$ and $1<p<\infty$.

$$
\left\|\nabla_{x}^{2} \int_{\mathbb{R}_{+}^{n}} N(\cdot-y) f(y) d y\right\|_{\dot{H}_{p}^{\alpha}} \leq c\|f\|_{\dot{H}_{p}^{\alpha}} .
$$

The proof of Lemma3.1 is given in Appendix $\mathrm{A}$.

3.2. Gaussian kernel. The fundamental solution of the heat equation in $\mathbb{R}^{n}$ is denoted by

$$
\Gamma(x, t)= \begin{cases}\frac{1}{(2 \pi t)^{\frac{n}{2}}} e^{-\frac{|x|^{2}}{4 t}} & \text { if } t>0, \\ 0 & \text { if } t \leq 0 .\end{cases}
$$

Let $\Gamma^{*}(x-y, t):=\Gamma\left(x^{\prime}-y^{\prime}, x_{n}+y_{n}, t\right)$. For $u_{0} \in B_{p \infty, 0}^{\alpha}, 1 \leq p \leq \infty, \alpha \in \mathbb{R}$, we define $\Gamma_{t} * u_{0 j}$ and $\Gamma_{t}^{*} * u_{0 j}$ by

$$
\begin{aligned}
& \Gamma_{t} * u_{0 j}(x):=\int_{\mathbb{R}_{+}^{n}} \Gamma(x-y, t) u_{0 j}(y) d y, \quad \Gamma_{t}^{*} * u_{0 j}(x):=\int_{\mathbb{R}_{+}^{n}} \Gamma^{*}(x-y, t) u_{0 j}(y) d y \quad \alpha \geq 0, \\
& \Gamma_{t} * u_{0 j}(x):=<u_{0}, \Gamma(x-\cdot, t)>, \quad \Gamma_{t}^{*} * u_{0 j}(x):=<u_{0}, \Gamma^{*}(x-\cdot, t)>\quad \alpha<0,
\end{aligned}
$$

where $<\cdot, \cdot>$ is dual paring between $\dot{B}_{p \infty}^{\alpha}$ and $\dot{B}_{p^{\prime} 1,0}^{-\alpha}$. 
Lemma 3.2. (1) Let $1 \leq p \leq \infty$ and $\alpha>0$. Let $u_{0} \in \dot{B}_{p \infty, 0}^{-\alpha}$ with $\operatorname{div} u_{0}=0$. Then,

$$
\left\|\Gamma_{t} * u_{0}\right\|_{L_{\frac{1}{2} \alpha}^{\infty} L^{p}},\left\|\Gamma_{t}^{*} * u_{0}\right\|_{L_{\frac{1}{2} \alpha}^{\infty} L^{p}} \leq c\left\|u_{0}\right\|_{\dot{B}_{p \infty}^{-\alpha}}
$$

(2) Let $1 \leq p \leq \infty$ and $\alpha>0$. Let $u_{0} \in \dot{B}_{p \infty, 0}^{\alpha}$ with $\operatorname{div} u_{0}=0$. Then,

$$
\left\|\Gamma_{t} * u_{0}\right\|_{L^{\infty} \dot{B}_{p \infty}^{\alpha}},\left\|\Gamma_{t}^{*} * u_{0}\right\|_{L^{\infty} \dot{B}_{p \infty}^{\alpha}} \leq c\left\|u_{0}\right\|_{\dot{B}_{p \infty}^{\alpha}}
$$

Proof. The proof of Lemma 3.2 is given in Appendix $\mathrm{B}$.

We define $\Gamma * f$ and $\Gamma^{*} * f$ by

$$
\begin{aligned}
\Gamma * f(x, t) & :=\int_{0}^{t} \int_{\mathbb{R}_{+}^{n}} \Gamma(x-y, t-s) f(y, s) d y d s, \\
\Gamma^{*} * f(x, t) & :=\int_{0}^{t} \int_{\mathbb{R}_{+}^{n}} \Gamma^{*}(x-y, t-s) f(y, s) d y d s .
\end{aligned}
$$

Lemma 3.3. (1) Let $1<p_{1} \leq p<\infty$ and $\alpha>0$ satisfying $-1+\alpha<\frac{n}{p_{1}}-\frac{n}{p}<1$. Let $f=$ div $\mathcal{F}$ with $\mathcal{F} \in L_{\frac{1}{2} \alpha+\frac{1}{2}-\frac{n}{2}\left(\frac{1}{p_{1}}-\frac{1}{p}\right)}^{\infty}\left(0, \infty, L^{p_{1}}\left(\mathbb{R}_{+}^{n}\right)\right)$. Then,

$\|\Gamma * \mathbb{P} f\|_{L_{\frac{1}{2} \alpha}^{\infty}\left(0, \infty ; L^{p}\left(\mathbb{R}_{+}^{n}\right)\right)},\left\|\Gamma^{*} * \mathbb{P} f\right\|_{L_{\frac{1}{2} \alpha}^{\infty}\left(0, \infty ; L^{p}\left(\mathbb{R}_{+}^{n}\right)\right)} \leq c\|\mathcal{F}\|_{L_{\frac{1}{2} \alpha+\frac{1}{2}-\frac{n}{2}\left(\frac{1}{p_{1}}-\frac{1}{p}\right)}^{\infty}\left(0, \infty ; L^{\left.p_{1}\left(\mathbb{R}_{+}^{n}\right)\right)}\right.}$.

(2) Let $0<\alpha<2$ and $1 \leq p_{1} \leq p$ such that $1+\frac{n-1}{p}<\frac{n}{p_{1}}<1+\frac{n}{p}$. Let $f=\operatorname{div} \mathcal{F}$ with $\mathcal{F} \in L_{\frac{1}{2}-\frac{n}{2 p_{1}}+\frac{n}{2 p}}^{\infty}\left(0, \infty ; \dot{B}_{p_{1} \infty}^{\alpha}\left(\mathbb{R}_{+}^{n}\right)\right)$. Then,

$\|\Gamma * \mathbb{P} f\|_{L^{\infty}\left(0, \infty ; \dot{B}_{p \infty}^{\alpha}\left(\mathbb{R}_{+}^{n}\right)\right)},\left\|\Gamma^{*} * \mathbb{P} f\right\|_{L^{\infty}\left(0, \infty ; \dot{B}_{p \infty}^{\alpha}\left(\mathbb{R}_{+}^{n}\right)\right)} \leq c\|\mathcal{F}\|_{L_{\frac{1}{2}-\frac{n}{2 p_{1}}+\frac{n}{2 p}}}\left(0, \infty ; \dot{B}_{p_{1} \infty}^{\alpha}\left(\mathbb{R}_{+}^{n}\right)\right)$.

Proof. The proof of Lemma 3.3 is given in Appendix C.

3.3. Hölder type inequality. The following Hölder type inequality is a well-known result (see Lemma 2.2 in [5]).

Lemma 3.4. Let $0<\beta$ and $1 \leq p, q \leq \infty$. Then, for $\frac{1}{r_{i}}+\frac{1}{s_{i}}=\frac{1}{p}, i=1,2$,

$$
\left\|f_{1} f_{2}\right\|_{\dot{B}_{p q}^{\beta}} \leq c\left(\left\|f_{1}\right\|_{\dot{B}_{s_{1} q}^{\beta}}\left\|f_{2}\right\|_{L^{r_{1}}}+\left\|f_{1}\right\|_{L^{s_{2}}}\left\|f_{2}\right\|_{\dot{B}_{r_{2} q}^{\beta}}\right)
$$

3.4. Helmholtz projection $\mathbb{P}$. Let $\mathbb{P}$ be a Helmholtz projection operator in $\mathbb{R}_{+}^{n}$. Let $f=\operatorname{div} \mathcal{F}, \mathcal{F}=$ $\left(F_{k l}\right)_{k, l=1}^{n}, F_{k l}=F_{l k}$, with $\left.F_{m k}\right|_{x_{n}=0}=0$. Then $\mathbb{P} f$ can be rewritten as $\mathbb{P} f=\operatorname{div} \mathcal{F}^{\prime}, \mathcal{F}^{\prime}=$ 
$\left(F_{k m}^{\prime}\right)_{k, m=1}^{n}$, where

$$
\begin{aligned}
F_{n m}^{\prime}= & F_{n m}-\delta_{n m} F_{n n}, m=1, \cdots, n, \\
F_{\beta \gamma}^{\prime}= & F_{\beta \gamma}-\delta_{\beta \gamma} F_{n n}+D_{x_{\gamma}}\left(\sum_{q=1}^{n} \int_{\mathbb{R}_{+}^{n}} D_{y_{q}} N^{+}(x, y) F_{\beta q}(y) d y\right. \\
& \left.+\int_{\mathbb{R}_{+}^{n}}\left(D_{y_{n}} N^{+}(x, y) F_{n \beta}(y)-D_{y_{\beta}} N^{+}(x, y) F_{n n}(y)\right) d y\right) \quad \beta, \gamma \neq n, \\
F_{\beta n}^{\prime}= & -\sum_{\gamma=1}^{n-1} D_{x_{\gamma}} \int_{\mathbb{R}_{+}^{n}} D_{x_{n}} N^{+}(x, y) F_{\beta \gamma}(y) d y+D_{x_{\beta}} \int_{R_{+}} D_{x_{n}} N^{+}(x, y) F_{n n}(y) d y \\
& -2 F_{\beta n}(x)-2 \sum_{\gamma=1}^{n-1} D_{x_{\gamma}} \int_{\mathbb{R}_{+}^{n}} D_{x_{\gamma}} N^{-}(x, y) F_{\beta n}(y) d y \quad \beta \neq n .
\end{aligned}
$$

(See Section 3 of [12] for the details). Here $N^{+}(x, y):=N(x-y)+N\left(x-y^{*}\right)$ and $N^{-}(x, y)=$ $N(x-y)-N\left(x-y^{*}\right)$. From Lemma 3.1 and real interpolation, we have

$$
\begin{aligned}
&\left\|\mathcal{F}^{\prime}\right\|_{\dot{H}_{p}^{\alpha}} \leq c\|\mathcal{F}\|_{\dot{H}_{p}^{\alpha}} 0 \leq \alpha, 1<p<\infty, \\
&\left\|\mathcal{F}^{\prime}\right\|_{\dot{B}_{p \infty}^{\alpha}} \leq c\|\mathcal{F}\|_{\dot{B}_{p \infty}^{\alpha}} \quad 0<\alpha, 1<p<\infty .
\end{aligned}
$$

\section{Proof of Theorem 1.3}

First, we decompose the Stokes equations (1.2) as the following two equations:

$$
\begin{aligned}
& v_{t}-\Delta v+\nabla \pi=0, \operatorname{div} v=0 \quad \text { in } \quad \mathbb{R}_{+}^{n} \times(0, \infty), \\
&\left.v\right|_{t=0}=u_{0} \quad \text { and }\left.\quad v\right|_{x_{n}=0}=0,
\end{aligned}
$$

and

$$
\begin{gathered}
V_{t}-\Delta V+\nabla \Pi=\operatorname{div} \mathcal{F}, \quad \operatorname{div} V=0 \text { in } \mathbb{R}_{+}^{n} \times(0, \infty), \\
\left.V\right|_{t=0}=0,\left.\quad V\right|_{x_{n}=0}=0 .
\end{gathered}
$$

Let $u=V+v$ and $p=\pi+\Pi$. Then, $(u, p)$ is a solution of (1.2).

4.1. Estimate of $(v, \pi)$. The solution $(v, \pi)$ of (4.1) is represented by (see [27])

$$
\begin{aligned}
v_{i}(x, t) & =\int_{\mathbb{R}_{+}^{n}} G_{i j}(x, y, t) u_{0 j}(y) d y, \\
\pi(x, t) & =\int_{\mathbb{R}_{+}^{n}} P(x, y, t) \cdot u_{0}(y) d y,
\end{aligned}
$$

where $G$ and $P$ are defined by

$$
\begin{aligned}
G_{i j}=\delta_{i j} & \left(\Gamma(x-y, t)-\Gamma\left(x-y^{*}, t\right)\right) \\
& +4\left(1-\delta_{j n}\right) \frac{\partial}{\partial x_{j}} \int_{0}^{x_{n}} \int_{\mathbb{R}^{n-1}} \frac{\partial N(x-z)}{\partial x_{i}} \Gamma\left(z-y^{*}, t\right) d z,
\end{aligned}
$$




$$
\begin{aligned}
P_{j}(x, y, t)= & 4\left(1-\delta_{j n}\right) \frac{\partial}{\partial x_{j}}\left[\int_{\mathbb{R}^{n-1}} \frac{\partial N\left(x^{\prime}-z^{\prime}, x_{n}\right)}{\partial x_{n}} \Gamma\left(z^{\prime}-y^{\prime}, y_{n}, t\right) d z^{\prime}\right. \\
& \left.+\int_{\mathbb{R}^{n-1}} N\left(x^{\prime}-z^{\prime}, x_{n}\right) \frac{\partial \Gamma\left(z^{\prime}-y^{\prime}, y_{n}, t\right)}{\partial y_{n}} d z^{\prime}\right] .
\end{aligned}
$$

From proofs of Lemma 3.1 and Lemma 3.3 in [10], we get

$$
\begin{aligned}
\|v(t)\|_{\dot{H}_{p}^{k}} \leq c\left(\left\|\Gamma_{t} * u_{0}\right\|_{\dot{H}_{p}^{k}}+\left\|\Gamma_{t}^{*} * u_{0}\right\|_{\dot{H}_{p}^{k}}\right) \quad 1<p<\infty, \quad k \in \mathbb{N} \cup\{0\} \\
\|v(t)\|_{\dot{B}_{p \infty}^{\alpha}} \leq c\left(\left\|\Gamma_{t} * u_{0}\right\|_{\dot{B}_{p \infty}^{\alpha}}+\left\|\Gamma_{t}^{*} * u_{0}\right\|_{\dot{B}_{p \infty}^{\alpha}}\right) \quad 1<p<\infty, \quad \alpha>0 .
\end{aligned}
$$

4.2. Estimate of $(V, \Pi)$. Let $\mathbb{P} f$ be the Helmholtz projection of $f$. Note that $\operatorname{div} \mathbb{P} f=0$ and $\left.(\mathbb{P} f)_{n}\right|_{x_{n}=0}=0$. We define $\left(V, \Pi_{0}\right)$ by

$$
\begin{aligned}
& V_{i}(x, t)=\int_{0}^{t} \int_{\mathbb{R}_{+}^{n}} G_{i j}(x, y, t-\tau)(\mathbb{P} f)_{j}(y, \tau) d y d \tau, \\
& \Pi_{0}(x, t)=\int_{0}^{t} \int_{\mathbb{R}_{+}^{n}} P(x, y, t-\tau) \cdot(\mathbb{P} f)(y, \tau) d y d \tau,
\end{aligned}
$$

where $G$ and $P$ are defined by (4.5) and (4.6). Then $\left(V, \Pi_{0}\right)$ satisfies

$$
\begin{gathered}
V_{t}-\Delta V+\nabla \Pi_{0}=\mathbb{P} f, \quad \operatorname{div} V=0, \text { in } \mathbb{R}_{+}^{n} \times(0, \infty), \\
\left.V\right|_{t=0}=0,\left.\quad V\right|_{x_{n}=0}=0 .
\end{gathered}
$$

(See [27].) Let $\Pi=\Pi_{0}+\mathbb{Q} f$. Then, $(V, \Pi)$ is the solution of [4.2).

Let $1<p<\infty, 1 \leq q \leq \infty$, and $0 \leq \alpha \leq 2$. In Section 3 in [10], the authors showed that $V$ defined by (4.8) has the following estimates (using real interpolations);

$$
\begin{aligned}
\|V(t)\|_{\dot{H}_{p}^{k}} & \leq c\left(\|\Gamma * \mathbb{P} f(t)\|_{\dot{H}_{p}^{k}}+\left\|\Gamma^{*} * \mathbb{P} f(t)\right\|_{\dot{H}_{p}^{k}}\right) \quad 1<p<\infty, k \in \mathbb{N} \cup\{0\}, \\
\|V(t)\|_{\dot{B}_{p \infty}^{\alpha}} & \leq c\left(\|\Gamma * \mathbb{P} f(t)\|_{\dot{B}_{p \infty}^{\alpha}}^{\alpha}+\left\|\Gamma^{*} * \mathbb{P} f(t)\right\|_{\dot{B}_{p \infty}^{\alpha}}\right) \quad 1<p<\infty, \alpha>0 .
\end{aligned}
$$

4.3. Estimate of $(u, p)$. Note that $(u, p)$ defined by $u=V+v$ and $p=\pi+\Pi_{0}+\mathbb{Q} \operatorname{div} \mathcal{F}$ is the solution of (1.2). From (4.7), (4.10), Lemma 3.2 and Lemma 3.3, we obtain Theorem 1.3,

\section{NONLINEAR PROBLEM}

In this section, we would like to give proof of Theorem 1.1. For this purpose, we construct approximate velocities and then derive uniform convergence in $L^{\infty} \dot{B}_{p \infty}^{\alpha}$.

5.1. Approximating solutions. Let $\left(u^{1}, p^{1}\right)$ be the solution of the Stokes equations

$$
\begin{gathered}
u_{t}^{1}-\Delta u^{1}+\nabla p^{1}=0, \quad \operatorname{div} u^{1}=0, \text { in } \mathbb{R}_{+}^{n} \times(0, \infty), \\
\left.u^{1}\right|_{t=0}=u_{0},\left.\quad u^{1}\right|_{x_{n}=0}=0 .
\end{gathered}
$$


Let $m \geq 1$. After obtaining $\left(u^{1}, p^{1}\right), \cdots,\left(u^{m}, p^{m}\right)$ construct $\left(u^{m+1}, p^{m+1}\right)$ which satisfies the following equations

$$
\begin{gathered}
u_{t}^{m+1}-\Delta u^{m+1}+\nabla p^{m+1}=f^{m}, \quad \operatorname{div} u^{m+1}=0, \text { in } \mathbb{R}_{+}^{n} \times(0, \infty), \\
\left.u^{m+1}\right|_{t=0}=u_{0},\left.\quad u^{m+1}\right|_{x_{n}=0}=0,
\end{gathered}
$$

where $f^{m}=-\operatorname{div}\left(u^{m} \otimes u^{m}\right)$.

5.2. Uniform boundedness in $L_{\frac{1}{2}-\frac{n}{2 p_{0}}}^{\infty} L^{p_{0}}, n<p_{0}$. From (1) of Theorem 1.3 , we have

$$
\left\|u^{1}\right\|_{\frac{1}{2}-\frac{n}{2 p_{0}}} L^{p_{0}} \leq c_{0}\left\|u_{0}\right\|_{\dot{B}_{p_{0} \infty, 0}^{-1+n / p_{0}}}:=N_{0} .
$$

From (1) of Theorem 1.3, taking $p_{1}=\frac{p_{0}}{2}$, we have

$$
\begin{aligned}
\left\|u^{m+1}\right\|_{L_{\frac{1}{2}-\frac{n}{2 p_{0}}}^{\infty}} L^{p_{0}} & \leq c\left(\left\|u_{0}\right\|_{\dot{B}_{p_{0} \infty, 0}^{-1+n / p_{0}}}+\left\|u^{m} \otimes u^{m}\right\|_{L_{1-\frac{n}{p_{0}}}^{\infty} L^{p_{0} / 2}}\right) \\
& \leq c_{1}\left(\left\|u_{0}\right\|_{\dot{B}_{p_{0} \infty, 0}^{-1+n / p_{0}}}+\left\|u^{m}\right\|_{L_{\frac{1}{2}-\frac{n}{2 p_{0}}}^{\infty}} L^{p_{0}}\right) .
\end{aligned}
$$

Under the hypothesis $\left\|u^{m}\right\|_{L_{\frac{1}{2}-\frac{n}{2 p_{0}}}^{\infty}} L^{p_{0}} \leq M_{0}$, (5.4) leads to the estimate

$$
\left\|u^{m+1}\right\|_{L_{\frac{1}{2}-\frac{n}{2 p_{0}}}^{\infty}} L^{p_{0}} \leq c_{1}\left(N_{0}+M_{0}^{2}\right) .
$$

Choose $M_{0}$ and $N_{0}$ so small that

$$
M_{0} \leq \frac{1}{2 c_{1}} \text { and } N_{0}<\frac{M_{0}}{2 c_{1}} .
$$

By the mathematical induction argument, we conclude

$$
\left\|u^{m}\right\|_{\frac{1}{2}-\frac{n}{2 p_{0}}} L^{p_{0}} \leq M_{0} \text { for all } m=1,2 \cdots .
$$

5.3. Uniform boundedness in $L^{\infty} \dot{B}_{p \infty}^{-1+n / p}$. From (2) of Theorem 1.3 , we have

$$
\left\|u^{1}\right\|_{L^{\infty} \dot{B}_{p \infty}^{-1+n / p}} \leq c_{2}\left\|u_{0}\right\|_{\dot{B}_{p \infty, 0}^{-1+n / p}}:=N
$$

We take $1 \leq p_{1}<p$ satisfying $1+\frac{n-1}{p}<\frac{n}{p_{1}}<1+\frac{n}{p}$. From (2) of Theorem 1.3 to obtain

$$
\left\|u^{m+1}\right\|_{L^{\infty} \dot{B}_{p \infty}^{-1+n / p}} \leq c\left(\left\|u_{0}\right\|_{\dot{B}_{p \infty, 0}^{-1+n / p}}+\left\|u^{m} \otimes u^{m}\right\|_{L_{\frac{1}{2}-\frac{n}{2 p_{1}}+\frac{n}{2 p}}^{\infty} \dot{B}_{p_{1} \infty}^{-1+n / p}}\right) .
$$

Let $\frac{1}{p_{1}}=\frac{1}{p_{0}}+\frac{1}{p}$ such that $1-\frac{1}{p}<\frac{n}{p_{0}}<1$. By Lemma 3.4, we have

$$
\begin{aligned}
\left\|\left(u^{m} \otimes u^{m}\right)\right\|_{L_{\frac{1}{2}-\frac{n}{2 p_{1}}+\frac{n}{2 p}}^{\infty} \dot{B}_{p_{1} \infty}^{-1+n / p}} & \leq c\left\|u^{m}\right\|_{L^{\infty} \dot{B}_{p \infty}^{-1+n / p}}\left\|u^{m}\right\|_{L_{\frac{1}{2}-\frac{n}{2 p_{0}}}^{\infty}} L^{p_{0}} \\
& \leq c\left\|u^{m}\right\|_{L^{\infty} \dot{B}_{p \infty}^{-1+n / p}}\left\|u^{m}\right\|_{L_{\frac{1}{2}-\frac{n}{2 p_{0}}}^{\infty}} L^{p_{0}} .
\end{aligned}
$$

From (5.8)-(5.9), we have

$$
\left\|u^{m+1}\right\|_{L^{\infty} \dot{B}_{p \infty}^{-1+n / p}} \leq c_{1}\left(N+\left\|u^{m}\right\|_{L_{\frac{1}{2}-\frac{n}{2 p_{0}}}^{\infty}} L^{p_{0}}\left\|u^{m}\right\|_{L^{\infty} \dot{B}_{p \infty}^{-1+n / p}}\right) .
$$


Under the hypothesis $\left\|u^{m}\right\|_{L^{\infty} \dot{B}_{p \infty}^{-1+n / p}} \leq M$, (5.10) leads to the estimate

$$
\left\|u^{m+1}\right\|_{L^{\infty} \dot{B}_{p \infty}^{-1+n / p}} \leq c_{1}\left(N+M_{0} M\right) .
$$

Choose that $M_{0}$ is small and $M$ is large so that that

$$
M_{0} \leq \frac{1}{2 c_{1}}, \quad 2 c_{1} N \leq M
$$

By the mathematical induction argument, we conclude

$$
\left\|u^{m}\right\|_{L^{\infty} \dot{B}_{p \infty}^{-1+n / p}} \leq M \text { for all } m=1,2 \cdots
$$

5.4. Uniform convergence. Let $U^{m}=u^{m+1}-u^{m}$ and $P^{m}=p^{m+1}-p^{m}$. Then, $\left(U^{m}, P^{m}\right)$ satisfy the equations

$$
\begin{gathered}
U_{t}^{m}-\Delta U^{m}+\nabla P^{m}=-\operatorname{div}\left(u^{m} \otimes U^{m-1}+U^{m-1} \otimes u^{m-1}\right), \quad \operatorname{div} U^{m}=0, \text { in } \mathbb{R}_{+}^{n} \times(0, \infty), \\
\left.U^{m}\right|_{t=0}=0,\left.\quad U^{m}\right|_{x_{n}=0}=0 .
\end{gathered}
$$

Recall the uniform estimates (5.6) and (5.12) for the approximate solutions. From Theorem 1.3 and Lemma 3.4 we have

$$
\begin{aligned}
\left\|U^{m}\right\|_{L_{\frac{1}{2}-\frac{n}{2 p_{0}}}^{\infty}} L^{p_{0}} & \leq c\left(\left\|u^{m-1}\right\|_{L_{\frac{1}{2}-\frac{n}{2 p_{0}}}^{\infty} L^{p_{0}}}+\left\|u^{m}\right\|_{L_{\frac{1}{2}-\frac{n}{2 p_{0}}}^{\infty}} L^{p_{0}}\right)\left\|U^{m-1}\right\|_{L_{\frac{1}{2}-\frac{n}{2 p_{0}}}^{\infty} L^{p_{0}}} \\
& \leq c_{5} M_{0}\left\|U^{m-1}\right\|_{L_{\frac{1}{2}-\frac{n}{2 p_{0}}}^{\infty}} L^{p_{0}},
\end{aligned}
$$

and

$$
\begin{aligned}
\left\|U^{m}\right\|_{L^{\infty} \dot{B}_{p \infty}^{-1+n / p} \leq} \leq & c\left\|u^{m} \otimes U^{m-1}+U^{m-1} \otimes u^{m-1}\right\|_{L^{\infty} \dot{B}_{p_{1} \infty}^{-1+n / p}} \\
\leq & c\left(\left\|u^{m}\right\|_{L^{\infty} \dot{B}_{p \infty}^{-1+n / p}}+\left\|u^{m-1}\right\|_{L^{\infty} \dot{B}_{p \infty}^{-1+n / p}}\right)\left\|U^{m-1}\right\|_{L_{\frac{1}{2}-\frac{n}{2 p_{0}}}^{\infty}} L^{p_{0}} \\
& \left.\quad+c\left(\left\|u^{m}\right\|_{L_{\frac{1}{2}-\frac{n}{2 p_{0}}}^{\infty}} L^{p_{0}}+\left\|u^{m-1}\right\|_{L_{\frac{1}{2}-\frac{n}{2 p_{0}}}} L^{p_{0}}\right)\left\|U^{m-1}\right\|_{L^{\infty} \dot{B}_{p \infty}^{-1+n / p}}\right) \\
\leq & c_{6} M\left\|U^{m-1}\right\|_{L_{\frac{1}{2}-\frac{n}{2 p_{0}}}} L^{p_{0}}+c_{6} M_{0}\left\|U^{m-1}\right\|_{L^{\infty} \dot{B}_{p \infty}^{-1+n / p}} .
\end{aligned}
$$

We take the constant $c_{6}$ greater than $c_{5}$, that is,

$$
c_{6}>c_{5} .
$$

From (5.13), if $c_{5} M_{0}<1$, then $\sum_{m=1}^{\infty}\left\|U^{m}\right\|_{L_{\frac{1}{2}-\frac{n}{2 p_{0}}}^{\infty}} L^{p_{0}}$ converges, that is,

$$
\sum_{m=1}^{\infty} U^{m} \text { converges in } L_{\frac{1}{2}-\frac{n}{2 p_{0}}}^{\infty} L^{p_{0}} .
$$

Take $A>0$ satisfying $A\left(c_{6}-c_{5}\right) M_{0} \geq c_{6} M$. Then from (5.13) and (5.14) it holds that

$$
\begin{array}{r}
\left\|U^{m}\right\|_{L^{\infty} \dot{B}_{p \infty}^{-1+n / p}}+A\left\|U^{m}\right\|_{L_{\frac{1}{2}-\frac{n}{2 p_{0}}}^{\infty}} L^{p_{0}} \\
\leq c_{6} M_{0}\left(\left\|U^{m-1}\right\|_{\left.L^{\infty} \dot{B}_{p \infty}^{-1+n / p}+A\left\|U^{m-1}\right\|_{L_{\frac{1}{2}-\frac{n}{2 p_{0}}}^{\infty}} L^{p_{0}}\right)}\right.
\end{array}
$$


Again if $c_{6} M_{0}<1$, then $\sum_{m=1}^{\infty}\left(\left\|U^{m}\right\|_{L^{\infty} \dot{B}_{p \infty}^{-1+n / p}}+A\left\|U^{m}\right\|_{L_{\frac{1}{2}-\frac{n}{2 p_{0}}}^{\infty}} L^{p_{0}}\right)$ converges. This implies that $\sum_{m=1}^{\infty}\left\|U^{m}\right\|_{L^{\infty} \dot{B}_{p \infty}^{-1+n / p}}$ converges, that is,

$$
\sum_{m=1}^{\infty} U^{m} \text { converges in } L^{\infty} \dot{B}_{p \infty}^{-1+n / p} .
$$

Therefore, if $M_{0}$ satisfies the condition (5.11) with the additional conditions

$$
M_{0}<\frac{1}{c_{6}}
$$

then $u^{m}=u^{1}+\sum_{k=1}^{m} U^{k}$ converges to $u^{1}+\sum_{k=1}^{\infty} U^{k}$ in $L^{\infty} \dot{B}_{p \infty}^{-1+n / p} \cap L_{\frac{1}{2}-\frac{n}{2 p_{0}}}^{\infty} L^{p_{0}}$. Set $u:=$ $u^{1}+\sum_{k=1}^{\infty} U^{k}$.

5.5. Existence. In this section, we will show that $u$ satisfies weak formulation of the Navier-Stokes equations (1.1), that is, $u$ is a weak solution of the Navier-Stokes equations (1.1) with appropriate distribution $p$.

Let $u$ be the same one constructed in the previous Section. Because $u_{m} \rightarrow u$ in $L_{\frac{1}{2}-\frac{n}{2 p_{0}}}^{\infty} L^{p_{0}}$ by (5.6), we have

$$
\|u\|_{L_{\frac{1}{2}-\frac{n}{2 p_{0}}}^{\infty}} L^{p_{0}}, \quad\left\|u^{m}\right\|_{L_{\frac{1}{2}-\frac{n}{2 p_{0}}}^{\infty}} L^{p_{0}} \leq M_{0} .
$$

Let $\Phi \in C_{0}^{\infty}\left(\mathbb{R}_{+}^{n} \times[0, T)\right)$ with $\operatorname{div} \Phi=0$ for some $T>0$. Observe that $-\int_{0}^{\infty} \int_{\mathbb{R}_{+}^{n}} u^{m+1} \cdot \Delta \Phi d x d t=\int_{0}^{\infty} \int_{\mathbb{R}_{+}^{n}} u^{m+1} \cdot \Phi_{t}+\left(u^{m} \otimes u^{m}\right): \nabla \Phi d x d t+\int_{\mathbb{R}_{+}^{n}} u_{0} \cdot \Phi(x, 0) d x$.

Now, send $m$ to the infinity, then, $u^{m} \rightarrow u$ in $L_{\frac{1}{2}-\frac{n}{2 p_{0}}}^{\infty} L^{p_{0}}$. Then, we have

$$
\begin{aligned}
& \int_{0}^{T} \int_{\mathbb{R}_{+}^{n}}\left(u^{m+1}-u\right) \cdot \Delta \Phi d x d t \leq \int_{0}^{T}\left\|u^{m+1}-u\right\|_{L^{p_{0}}}\|\Delta \Phi\|_{L^{p_{0}^{\prime}}} d t \\
& \leq\left\|u^{m+1}-u\right\|_{L_{\frac{1}{2}-\frac{n}{2 p_{0}}}^{\infty}} L^{p_{0}} \int_{0}^{T} t^{-\frac{1}{2}+\frac{n}{2 p_{0}}}\|\Delta \Phi\|_{L^{p_{0}^{\prime}}} d t \\
& \rightarrow 0 \quad \text { as } m \rightarrow \infty \text {. }
\end{aligned}
$$

Similarly, we have

$$
\int_{0}^{T} \int_{\mathbb{R}_{+}^{n}}\left(u^{m+1}-u\right) \cdot \Phi_{t} d x d t \rightarrow 0 \quad \text { as } m \rightarrow \infty .
$$

Because $p_{0}>n$, we have

$$
\begin{aligned}
\int_{0}^{\infty} \int_{\mathbb{R}_{+}^{n}}\left(u^{m} \otimes\left(u^{m}-u\right)\right): \nabla \Phi d x d t & \leq\left\|u^{m} \otimes\left(u^{m+1}-u\right)\right\|_{L_{1-\frac{n}{p_{0}}}^{\infty}} L^{p_{0} / 2} \int_{0}^{T} t^{-1+\frac{n}{p_{0}}\|\Delta \Phi\|_{L^{\left(p_{0} / 2\right)^{\prime}}} d t} \\
& \leq\left\|u^{m}-u\right\|_{L_{\frac{1}{2}-\frac{n}{2 p_{0}}}^{\infty}} L^{p_{0}}\left\|u^{m}\right\|_{L_{\frac{1}{2}-\frac{n}{2 p_{0}}}^{\infty}} L^{p_{0}} \int_{0}^{T} t^{-1+\frac{n}{p_{0}}}\|\Delta \Phi\|_{L^{\left(p_{0} / 2\right)^{\prime}}} d t \\
& \rightarrow 0 \quad \text { as } m \rightarrow \infty .
\end{aligned}
$$


Hence, we have the identity

$$
-\int_{0}^{\infty} \int_{\mathbb{R}_{+}^{n}} u \cdot \Delta \Phi d x d t=\int_{0}^{\infty} \int_{\mathbb{R}_{+}^{n}} u \cdot \Phi_{t}+(u \otimes u): \nabla \Phi d x d t+\int_{\mathbb{R}_{+}^{n}} u_{0} \cdot \Phi(x, 0) d x .
$$

Therefore, $u$ is a weak solution of the Navier-Stokes equations (1.1). This completes the proof of the existence part of Theorem 1.1

5.6. Uniqueness in space $L_{\frac{1}{2}-\frac{n}{2 p_{0}}}^{\infty} L^{p_{0}}$. Let $u_{1} \in L_{\frac{1}{2}-\frac{n}{2 p_{0}}}^{\infty} L^{p_{0}}$ be another weak solution of the Navier-Stokes equations (1.1) with pressure $p_{1}$. Then, $\left(u-u_{1}, p-p_{1}\right)$ satisfies the equations

$$
\begin{gathered}
\left(u-u_{1}\right)_{t}-\Delta\left(u-u_{1}\right)+\nabla\left(p-p_{1}\right)=-\operatorname{div}\left(u \otimes\left(u-u_{1}\right)+\left(u-u_{1}\right) \otimes u_{1}\right) \text { in } \mathbb{R}_{+}^{n} \times(0, \infty), \\
\operatorname{div}\left(u-u_{1}\right)=0, \text { in } \mathbb{R}_{+}^{n} \times(0, \infty), \\
\left.\left(u-u_{1}\right)\right|_{t=0}=0,\left.\quad\left(u-u_{1}\right)\right|_{x_{n}=0}=0 .
\end{gathered}
$$

Applying the estimate of Theorem 1.3 to the above Stokes equations, we have

$$
\begin{aligned}
\| u- & u_{1}\left\|_{L_{\frac{1}{2}-\frac{n}{2 p_{0}}}^{\infty}} L^{p_{0}} \leq c\right\| u \otimes\left(u-u_{1}\right)+\left(u-u_{1}\right) \otimes u_{1} \|_{L_{1-\frac{n}{p_{0}}}^{\infty}} L^{p_{0}} \\
& \leq c_{5}\left(\|u\|_{L_{\frac{1}{2}-\frac{n}{2 p_{0}}}^{\infty}} L^{p_{0}}+\left\|u_{1}\right\|_{L_{\frac{1}{2}-\frac{n}{2 p_{0}}}^{\infty}} L^{p_{0}}\right)\left\|u-u_{1}\right\|_{L_{\frac{1}{2}-\frac{n}{2 p_{0}}}^{\infty}} L^{p_{0}} .
\end{aligned}
$$

Taking $M_{0}$ to satisfy $2 c_{5} M_{0}<1$, we have

$$
\left\|u-u_{1}\right\|_{L_{\frac{1}{2}-\frac{n}{2 p_{0}}}^{\infty}} L^{p_{0}}<\left\|u-u_{1}\right\|_{L_{\frac{1}{2}-\frac{n}{2 p_{0}}}^{\infty}} L^{p_{0}} .
$$

This implies that $u \equiv u_{1}$ in $\mathbb{R}_{+}^{n} \times(0, \infty)$ and so we complete the proof of the uniqueness part of Theorem 1.1.

\section{ApPendix A. Proof of Lemma 3.1}

The following lemma is well known trace theorem (see Theorem 6.6.1 in [3]).

Lemma A.1. Let $1<p<\infty$. If $f \in \dot{H}_{p}^{\alpha}$ for $\alpha>\frac{1}{p}$, then $\left.f\right|_{x_{n}=0} \in \dot{B}_{p p}^{\alpha-\frac{1}{p}}\left(\mathbb{R}^{n-1}\right)$ with

$$
\left\|\left.f\right|_{x_{n}=0}\right\|_{\dot{B}_{p p}^{\alpha-1 / p}\left(\mathbb{R}^{n-1}\right)} \leq c\|f\|_{\dot{H}_{p}^{\alpha}} .
$$

We define $N f$ by

$$
N f(x)=\int_{\mathbb{R}^{n-1}} N\left(x^{\prime}-y^{\prime}, x_{n}\right) f\left(y^{\prime}\right) d y^{\prime} .
$$

Observe that $D_{x_{n}} N f$ is the Poisson operator of the Laplace equation in $\mathbb{R}_{+}^{n}$ and $D_{x_{i}} N f=$ $D_{x_{n}} N R_{i}^{\prime} f$ for $i \neq n$, where $R^{\prime}=\left(R_{1}, \cdots, R_{n-1}\right)$ is the $n-1$ dimensional Riesz operator. The Poisson operator is bounded from $\dot{B}_{p p}^{\alpha-\frac{1}{p}}\left(\mathbb{R}^{n-1}\right)$ to $\dot{H}_{p}^{\alpha}\left(\mathbb{R}_{+}^{n}\right), \alpha \geq 0$ and $R^{\prime}$ is bounded from $\dot{B}_{p p}^{s}\left(\mathbb{R}^{n-1}\right)$ to $\dot{B}_{p p}^{s}\left(\mathbb{R}^{n-1}\right), s \in \mathbb{R}$ (see [30]). Hence the following estimates hold. 
Lemma A.2. Let $1<p<\infty$. Then

$$
\left\|\nabla_{x} N f\right\|_{\dot{H}_{p}^{\alpha}} \leq c\|f\|_{\dot{B}_{p p}^{\alpha-1 / p}\left(\mathbb{R}^{n-1}\right)} \quad \alpha \geq 0 .
$$

According to the Calderón-Zygmund inequality

$$
\left\|\int_{\mathbb{R}^{n}} \nabla_{x}^{2} N(\cdot-y) f(y) d y\right\|_{L^{p}} \leq c\|f\|_{L^{p}} \quad \text { for } \quad 1<p<\infty .
$$

We will show that for $k \geq 0$.

$$
\left\|D_{x}^{k} \nabla_{x}^{2} \int_{\mathbb{R}_{+}^{n}} N(x-y) f(y) d y(x)\right\|_{L^{p}} \leq c\left\|D_{x}^{k} f\right\|_{L^{p}} .
$$

Then, by the property of complex interpolation (see (2.1) and (2.2)), we get Lemma 3.1.

Note that

$$
\begin{aligned}
& D_{x_{i}} \int_{\mathbb{R}_{+}^{n}} N(x-y) f(y) d y=\int_{\mathbb{R}_{+}^{n}} N(x-y) D_{y_{i}} f(y) d y, i \neq n, \\
& D_{x_{n}} \int_{\mathbb{R}_{+}^{n}} N(x-y) f(y) d y=\int_{\mathbb{R}_{+}^{n}} N(x-y) D_{y_{n}} f(y) d y-\int_{\mathbb{R}^{n-1}} N\left(x^{\prime}-y^{\prime}, x_{n}\right) f\left(y^{\prime}, 0\right) d y^{\prime} .
\end{aligned}
$$

By (A.3), (A.2) and Lemma A.1, we have

$$
\begin{aligned}
\left\|\nabla_{x}^{3} \int_{\mathbb{R}_{+}^{n}} N(x-y) f(y) d y\right\|_{L^{p}} & \leq c\left(\left\|\nabla_{x} f\right\|_{L^{p}}+\|f(\cdot, 0)\|_{\dot{B}_{p p}^{1-\frac{1}{p}}\left(\mathbb{R}^{n-1}\right)}\right) \\
& \leq c\left\|\nabla_{x} f\right\|_{L^{p}} .
\end{aligned}
$$

By the successive argument, A.4 can be obtained for any multiple integer $k \geq 0$.

\section{ApPendix B. Proof of Lemma 3.2}

Fix a Schwartz function $\phi \in \mathcal{S}\left(\mathbb{R}^{n}\right)$ satisfying $\hat{\phi}(\xi)>0$ on $\frac{1}{2}<|\xi|<2, \hat{\phi}(\xi)=0$ elsewhere, and $\sum_{j=-\infty}^{\infty} \hat{\phi}\left(2^{-j} \xi\right)=1$ for $\xi \neq 0$. Let

$$
\widehat{\phi}_{j}(\xi):=\widehat{\phi}\left(2^{-j} \xi\right), \quad(j=0, \pm 1, \pm 2, \cdots),
$$

where $\hat{f}=\mathcal{F}(f)$ is a Fourier transform of $f$. Let $\Phi=\phi_{-1}+\phi_{0}+\phi_{1}$ and $\Phi_{j}(\xi)=\Phi\left(2^{-j} \xi\right)$ such that $\operatorname{supp} \Phi_{j} \subset\left\{2^{-j-2}<|\xi|<2^{-j+2}\right\}$ and $\Phi \equiv 1$ in $2^{j-1}<|\xi|<2^{j+1}$.

Lemma B.1. Let $\rho_{t j}(\xi)=\Phi_{j}(\xi) e^{-t|\xi|^{2}}$ for each integer $j$. Then $\rho_{t j}(\xi)$ are $L^{\infty}\left(\mathbb{R}^{n}\right)$-multipliers with the finite norm $M(t, j)$. Moreover for $t>0$

$$
M(t, j) \leq c e^{-\frac{1}{4} t 2^{2 j}} \sum_{0 \leq i \leq n} t^{i} 2^{2 i j} \leq c e^{-\frac{1}{8} t 2^{2 j}} .
$$

See Lemma 13 in [11].

Let $\tilde{u}_{0}(x)=u_{0}(x)$ be a zero extension over $\mathbb{R}^{n}$ such that $\left\|\tilde{u}_{0}\right\|_{\dot{B}_{p \infty}^{\alpha}\left(\mathbb{R}^{n}\right)} \leq c\left\|u_{0}\right\|_{\dot{B}_{p \infty}^{\alpha}}$. Let

$$
v(x, t)=\int_{\mathbb{R}^{n}} \Gamma(x-y, t) \tilde{u}_{0}(y) d y .
$$


Then, we have $\|v\|_{L^{\infty} \dot{B}_{p \infty}^{\alpha}} \leq c\|v\|_{L^{\infty}\left(0, \infty ; \dot{B}_{p \infty}^{\alpha}\left(\mathbb{R}^{n}\right)\right)}$.

Using the dyadic partition of unity $\sum_{j=-\infty}^{\infty} \hat{\phi}\left(2^{-j} \xi\right)=1$ for $\xi \neq 0$, we can write

$$
\mathcal{F}\left(v * \phi_{j}\right)(\xi, t)=\hat{\phi}\left(2^{-j} \xi\right) e^{-t|\xi|^{2}} \widehat{\tilde{u}_{0}}(\xi) .
$$

For $t>0$ we have

$$
\begin{aligned}
\left\|v * \phi_{j}(t)\right\|_{L^{p}\left(\mathbb{R}^{n}\right)} & =\left(\int_{\mathbb{R}^{n}}\left|\mathcal{F}^{-1}\left(e^{-t|\xi|^{2}} \hat{\phi}_{j}(\xi) \widehat{\tilde{u}_{0}}(\xi)\right)(x)\right|^{p} d x\right)^{\frac{1}{p}} \\
& =\left(\int_{\mathbb{R}^{n}}\left|\mathcal{F}^{-1}\left(\hat{\Phi}_{j}(\xi) e^{-t|\xi|^{2}} \hat{\phi}_{j}(\xi) \widehat{\tilde{u}_{0}}(\xi)\right)(x)\right|^{p} d x\right)^{\frac{1}{p}} .
\end{aligned}
$$

By Lemma B.1, we have

$$
\begin{aligned}
t^{\frac{1}{2} \alpha}\|v(t)\|_{L^{p}\left(\mathbb{R}^{n}\right)} & \leq t^{\frac{1}{2} \alpha} \sum_{-\infty<j<\infty}\left\|v(t) * \phi_{j}\right\|_{L^{p}\left(\mathbb{R}^{n}\right)} \\
& \leq t^{\frac{1}{2} \alpha} \sum_{-\infty<j<\infty} M(t, j)\left\|\tilde{u}_{0} * \phi_{j}\right\|_{L^{p}\left(\mathbb{R}^{n}\right)} \\
& \leq c t^{\frac{1}{2} \alpha} \sum_{-\infty<j<\infty} 2^{j \alpha} 2^{-t 2^{2 j}} 2^{-j \alpha}\left\|\tilde{u}_{0} * \phi_{j}\right\|_{L^{p}\left(\mathbb{R}^{n}\right)} \\
& \leq c t^{\frac{1}{2} \alpha} \sum_{-\infty<j<\infty} 2^{j \alpha} 2^{-t 2^{2 j}}\left\|\tilde{u}_{0}\right\|_{\dot{B}_{p \infty}^{-\alpha}\left(\mathbb{R}^{n}\right)} \\
& \leq c\left\|\tilde{u}_{0}\right\|_{\dot{B}_{p \infty}^{-\alpha}\left(\mathbb{R}^{n}\right)}
\end{aligned}
$$

and

$$
\begin{aligned}
2^{\alpha j}\left\|v(t) * \phi_{j}\right\|_{L^{p}\left(\mathbb{R}^{n}\right)} & \leq 2^{\alpha j} M(t, j)\left\|\tilde{u}_{0} * \phi_{j}\right\|_{L^{p}\left(\mathbb{R}^{n}\right)} \\
& \leq c 2^{\alpha j} 2^{-t 2^{2 j}}\left\|\tilde{u}_{0} * \phi_{j}\right\|_{L^{p}\left(\mathbb{R}^{n}\right)} \\
& \leq c\left\|\tilde{u}_{0}\right\|_{\dot{B}_{p \infty}^{\alpha}\left(\mathbb{R}^{n}\right)} .
\end{aligned}
$$

We complete the proof of Lemma 3.2.

\section{Appendix C. Proof of Lemma 3.3}

Because the proofs will be done in the same way, we prove only the case of $\Gamma^{*} * \mathbb{P} f$.

A crucial step in the proof of Lemma 3.3 is the following lemma, which is probably known to experts, however, we could not find it in the literature and thus, we provide its proof.

Lemma C.1. Let $X_{i}$ and $Y_{i}, i=1,2$ be Banach spaces and $0<t$ be fixed. Let $T: L^{1}\left(0, t ; X_{i}\right) \rightarrow$ $Y_{i}, i=1,2$ be linear operators such that

$$
\|T f\|_{Y_{i}} \leq M_{i} \int_{0}^{t}(t-s)^{-\beta_{i}}\|f(s)\|_{X_{i}} d s, \quad i=1,2 \quad \forall f \in L^{1}\left(0, t ; X_{i}\right) .
$$


Then, for $0<\theta<1$ and $1 \leq q \leq \infty$,

$$
\|T f\|_{\left(Y_{1}, Y_{2}\right)_{\theta, q}} \leq M_{1}^{\theta} M_{2}^{1-\theta} \int_{0}^{t}(t-s)^{-\beta}\|f(s)\|_{\left(X_{1}, X_{2}\right)_{\theta, q}} d s
$$

where $\beta=\beta_{1} \theta+\beta_{2}(1-\theta)$.

Proof. See Lemma C.1 in [9].

Lemma C.2. Let

$$
w(x, t)=\int_{0}^{t} \int_{\mathbb{R}^{n-1}} D_{x} \Gamma\left(x^{\prime}-y^{\prime}, x_{n}, t-\tau\right) f\left(y^{\prime}, \tau\right) d y^{\prime} d \tau .
$$

Then, for $\beta>0$,

$$
\|w(t)\|_{L^{p}} \leq c \int_{0}^{t}(t-\tau)^{\frac{1}{2 p}-1-\frac{\beta}{2}}\|f(\tau)\|_{\dot{B}_{p}^{-\beta}\left(\mathbb{R}^{n-1}\right)} d \tau .
$$

Proof. Let $D_{x}=D_{x_{n}}$. Then,

$$
\begin{aligned}
\|w(t)\|_{L^{p}} & \leq \int_{0}^{t}\left(\int_{0}^{\infty} \frac{x_{n}^{p}}{(t-\tau)^{\frac{3 p}{2}}} e^{-\frac{x_{n}^{2}}{t-\tau}} d x_{n}\right)^{\frac{1}{p}}\left\|\Gamma_{t-\tau}^{\prime} *^{\prime} f(\tau)\right\|_{L^{p}\left(\mathbb{R}^{n-1}\right)} d \tau \\
& =c \int_{0}^{t}(t-\tau)^{\frac{1}{2 p}-1}\left\|\Gamma_{t-\tau}^{\prime} *^{\prime} f(\tau)\right\|_{L^{p}\left(\mathbb{R}^{n-1}\right)} d \tau .
\end{aligned}
$$

Here, $\Gamma_{t}^{\prime}$ is a Gaussian kernel in $\mathbb{R}^{n-1}$ and $*^{\prime}$ is convolution in $\mathbb{R}^{n-1}$. Then, we have

$$
\begin{aligned}
\left\|\Gamma_{t-s}^{\prime} *^{\prime} f(\tau)\right\|_{L^{p}\left(\mathbb{R}^{n-1}\right)} & =\left\|\sum_{-\infty<k<\infty} \Phi_{k}^{\prime} *^{\prime} \Gamma_{t-s}^{\prime} *^{\prime} \phi_{k}^{\prime} *^{\prime} f(\tau)\right\|_{L^{p}\left(\mathbb{R}^{n-1}\right)} \\
& \leq \sum_{-\infty<k<\infty}\left\|\Phi_{k}^{\prime} *^{\prime} \Gamma_{t-s}^{\prime}\right\|_{M_{p}\left(\mathbb{R}^{n-1}\right)}\left\|\phi_{k}^{\prime} *^{\prime} f(\tau)\right\|_{L^{p}\left(\mathbb{R}^{n-1}\right)} \\
& \leq c \sum_{-\infty<k<\infty} e^{-(t-s) 2^{2 k}}\left\|\phi_{k}^{\prime} *^{\prime} f(\tau)\right\|_{L^{p}\left(\mathbb{R}^{n-1}\right)} \\
& \leq c\left(\sum_{-\infty<k<\infty} e^{-(t-s) \frac{p}{p-1} 2^{2 k}} 2^{\beta \frac{p}{p-1} k}\right)^{\frac{p-1}{p}}\left(\sum_{-\infty<k<\infty} 2^{-\beta p k}\left\|\phi_{k}^{\prime} *^{\prime} f(\tau)\right\|_{L^{p}\left(\mathbb{R}^{n-1}\right)}^{p}\right)^{\frac{1}{p}} \\
& \leq c(t-s)^{-\frac{\beta}{2}}\|f(\tau)\|_{\dot{B}_{p}^{-\beta}\left(\mathbb{R}^{n-1}\right)^{\prime}}
\end{aligned}
$$

From (C.2) and (C.3), we obtain (C.1).

Let $D_{x}=D_{x^{\prime}}$. Then,

$$
\begin{aligned}
\|w(t)\|_{L^{p}} & \leq \int_{0}^{t}\left(\int_{0}^{\infty} \frac{1}{(t-\tau)^{\frac{p}{2}}} e^{-\frac{x_{n}^{2}}{t-\tau}} d x_{n}\right)^{\frac{1}{p}}\left\|D_{x^{\prime}} \Gamma_{t-\tau}^{\prime} *^{\prime} f(\tau)\right\|_{L^{p}\left(\mathbb{R}^{n-1}\right)} d \tau \\
& =c \int_{0}^{t}(t-\tau)^{\frac{1}{2 p}-\frac{1}{2}}\left\|D_{x^{\prime}} \Gamma_{t-\tau}^{\prime} *^{\prime} f(\tau)\right\|_{L^{p}\left(\mathbb{R}^{n-1}\right)} d \tau
\end{aligned}
$$


Here, $\Gamma_{t}^{\prime}$ is a Gaussian kernel in $\mathbb{R}^{n-1}$ and $*^{\prime}$ is convolution in $\mathbb{R}^{n-1}$. Then, we have

$$
\begin{aligned}
\left\|D_{x_{n}} \Gamma_{t-s}^{\prime} *^{\prime} f(\tau)\right\|_{L^{p}\left(\mathbb{R}^{n-1}\right)} & \left\|\sum_{-\infty<k<\infty} D_{x^{\prime}} \Phi_{k}^{\prime} *^{\prime} \Gamma_{t-s}^{\prime} *^{\prime} \phi_{k}^{\prime} *^{\prime} f(\tau)\right\|_{L^{p}\left(\mathbb{R}^{n-1}\right)} \\
\leq & \sum_{-\infty<k<\infty}\left\|D_{x^{\prime}} \Phi_{k}^{\prime} *^{\prime} \Gamma_{t-s}^{\prime}\right\|_{M_{p}\left(\mathbb{R}^{n-1}\right)}\left\|\phi_{k}^{\prime} *^{\prime} f(\tau)\right\|_{L^{p}\left(\mathbb{R}^{n-1}\right)} \\
\leq & c \sum_{-\infty<k<\infty} 2^{k} e^{-(t-s) 2^{2 k}}\left\|\phi_{k}^{\prime} *^{\prime} f(\tau)\right\|_{L^{p}\left(\mathbb{R}^{n-1}\right)} \\
\leq & c\left(\sum_{-\infty<k<\infty} 2^{\frac{p}{p-1} k} e^{-(t-s) \frac{p}{p-1} 2^{2 k}} 2^{\beta \frac{p}{p-1} k}\right)^{\frac{p-1}{p}}\left(\sum_{-\infty<k<\infty} 2^{-\beta p k}\left\|\phi_{k}^{\prime} *^{\prime} f(\tau)\right\|_{L^{p}\left(\mathbb{R}^{n-1}\right)}^{p}\right)^{\frac{1}{p}} \\
& \leq c(t-s)^{-\frac{1}{2}-\frac{\beta}{2}}\|f(\tau)\|_{\dot{B}_{p}^{-\beta}\left(\mathbb{R}^{n-1}\right)}
\end{aligned}
$$

From (C.4) and (C.5), we obtain (C.1).

Proof of Lemma 3.3 Recalling Helmholtz decomposition of $f=\operatorname{divF}$ with $\left.F\right|_{x_{n}=0}=0$ in Section 3.4, we have

$$
\begin{aligned}
\Gamma^{*} *(\mathbb{P} f)_{j}(x, t) & =\int_{0}^{t} \int_{\mathbb{R}_{+}^{n}} \Gamma\left(x-y^{*}, t-\tau\right) \cdot \operatorname{div} F_{j}^{\prime}(y, \tau) d y d \tau \\
& =-\int_{0}^{t} \int_{\mathbb{R}_{+}^{n}} \nabla_{y} \Gamma\left(x-y^{*}, t-\tau\right) \cdot F_{j}^{\prime}(y, \tau) d y d \tau .
\end{aligned}
$$

Using (C.6), Young's inequality and (3.3), for $p_{1} \leq p$, we have

$$
\begin{aligned}
\left\|\Gamma^{*} *(\mathbb{P} f)(t)\right\|_{L^{p}} & \leq c \int_{0}^{t}(t-s)^{-\frac{1}{2}-\frac{n}{2}\left(\frac{1}{p_{1}}-\frac{1}{p}\right)} \sum_{j=1}^{j=n}\left\|F_{j}^{\prime}(s)\right\|_{L^{p_{1}}} d s \\
& \leq c \int_{0}^{t}(t-s)^{-\frac{1}{2}-\frac{n}{2}\left(\frac{1}{p_{1}}-\frac{1}{p}\right)}\|\mathcal{F}(s)\|_{L^{p_{1}}} d s .
\end{aligned}
$$

Similarly, we get

$$
\begin{aligned}
\left\|\nabla \Gamma^{*} *(\mathbb{P} f)(t)\right\|_{L^{p}} & \leq c \int_{0}^{t}(t-s)^{-\frac{1}{2}-\frac{n}{2}\left(\frac{1}{p_{1}}-\frac{1}{p}\right)} \sum_{j=1}^{n}\left\|\nabla F_{j}^{\prime}(s)\right\|_{L^{p_{1}}} d s \\
& \leq c \int_{0}^{t}(t-s)^{-\frac{1}{2}-\frac{n}{2}\left(\frac{1}{p_{1}}-\frac{1}{p}\right)}\|\mathcal{F}(s)\|_{\dot{H}_{p_{1}}^{1}} d s .
\end{aligned}
$$

From (C.6), we have

$$
\begin{aligned}
\nabla_{x}^{2} \Gamma^{*} *(\mathbb{P} f)_{j}(x, t)= & -\int_{0}^{t} \int_{\mathbb{R}_{+}^{n}} \nabla_{y} \Gamma\left(x-y^{*}, t-\tau\right) \cdot \nabla_{y}^{2} F_{j}^{\prime}(y, \tau) d y d \tau \\
& +\int_{0}^{t} \int_{\mathbb{R}^{n-1}} D_{x} \Gamma\left(x^{\prime}-y^{\prime}, x_{n}, t-s\right) D_{y_{n}} F_{j}\left(y^{\prime}, 0, s\right) d y^{\prime} d \tau
\end{aligned}
$$


Hence from Lemma C.2, for $\beta>0$, we have

$$
\begin{aligned}
\left\|D_{x}^{2} \Gamma^{*} *(\mathbb{P} f)(t)\right\|_{L^{p}} \leq & \int_{0}^{t}(t-\tau)^{-\frac{1}{2}-\frac{n}{2 p_{1}}+\frac{n}{2 p}}\left\|D_{y}^{2} F_{j}^{\prime}(\tau)\right\|_{L^{p_{1}\left(\mathbb{R}_{+}^{n}\right)}} d \tau \\
& +\int_{0}^{t}(t-\tau)^{\frac{1}{2 p}-1-\frac{\beta}{2}}\left\|D_{y} F_{j}^{\prime}(\tau)\right\|_{\dot{B}_{p}^{-\beta}\left(\mathbb{R}^{n-1}\right)} d \tau \\
:= & I_{1}(t)+I_{2}(t) .
\end{aligned}
$$

From (3.3), we have

$$
I_{1}(t) \leq c \int_{0}^{t}(t-\tau)^{-\frac{1}{2}-\frac{n}{2 p_{1}}+\frac{n}{2 p}}\|F(\tau)\|_{\dot{H}_{p_{1}}^{2}} d \tau
$$

Because $1+\frac{n-1}{p}<\frac{n}{p_{1}}<1+\frac{n}{p}$, taking $\beta=-1+\frac{n}{p_{1}}-\frac{n-1}{p}>0$, we have

$$
\begin{aligned}
I_{2}(t) & \leq c \int_{0}^{t}(t-\tau)^{\frac{1}{2 p}-1-\frac{\beta}{2}}\left\|D_{y} F_{j}^{\prime}(\tau)\right\|_{\dot{B}_{p_{1}}^{1-\frac{1}{p_{1}}}\left(\mathbb{R}^{n-1}\right)} d \tau \\
& \leq c \int_{0}^{t}(t-\tau)^{\frac{1}{2 p}-1-\frac{\beta}{2}}\left\|D_{y} F_{j}^{\prime}(\tau)\right\|_{\dot{H}_{p_{1}}^{1}\left(\mathbb{R}_{+}^{n}\right)} d \tau \\
& \leq c \int_{0}^{t}(t-\tau)^{-\frac{1}{2}-\frac{n}{2}\left(\frac{1}{p_{1}}-\frac{1}{p}\right)}\|F(\tau)\|_{\dot{H}_{p_{1}}^{2}\left(\mathbb{R}_{+}^{n}\right)} d \tau .
\end{aligned}
$$

Hence, we obtain

$$
\left\|D_{x}^{2} \Gamma^{*} *(\mathbb{P} f)(t)\right\|_{L^{p}} \leq c \int_{0}^{t}(t-\tau)^{-\frac{1}{2}-\frac{n}{2 p_{1}}+\frac{n}{2 p}}\|F(\tau)\|_{\dot{H}_{p_{1}}^{2}} d \tau .
$$

Then, from (C.7), we get

$$
\begin{aligned}
\left\|\Gamma^{*} *(\mathbb{P} f)(t)\right\|_{L^{p}} & \leq c \int_{0}^{t}(t-s)^{-\frac{1}{2}-\frac{n}{2}\left(\frac{1}{p_{1}}-\frac{1}{p}\right)}\|\mathcal{F}(s)\|_{L^{p_{1}}} d s \\
& \leq c \sup _{0<s<t}\left(s^{\frac{1}{2} \alpha+\frac{1}{2}-\frac{n}{2}\left(\frac{1}{p_{1}}-\frac{1}{p}\right)}\|\mathcal{F}(s)\|_{L^{p_{1}}}\right) \int_{0}^{t}(t-s)^{-\frac{1}{2}-\frac{n}{2}\left(\frac{1}{p_{1}}-\frac{1}{p}\right)} s^{-\frac{1}{2} \alpha-\frac{1}{2}+\frac{n}{2}\left(\frac{1}{p_{1}}-\frac{1}{p}\right)} d s \\
& =c t^{-\frac{1}{2} \alpha} \sup _{0<s<t}\left(s^{\frac{1}{2} \alpha+\frac{1}{2}-\frac{n}{2}\left(\frac{1}{p_{1}}-\frac{1}{p}\right)}\|\mathcal{F}(s)\|_{L^{p_{1}}}\right) .
\end{aligned}
$$

Hence, we complete the proof of (1) of Lemma 3.3 .

From (C.7), (C.8), Lemma C.1 and (2.3), for $0<\alpha<1$ and $\frac{n}{p_{1}}<1+\frac{n}{p}$, we get

$$
\begin{aligned}
\left\|\Gamma^{*} *(\mathbb{P} f)(t)\right\|_{\dot{B}_{p \infty}^{\alpha}} & \leq c \int_{0}^{t}(t-s)^{-\frac{1}{2}-\frac{n}{2}\left(\frac{1}{p_{1}}-\frac{1}{p}\right)}\|\mathcal{F}(s)\|_{\dot{B}_{p_{1} \infty}^{\alpha}} d s \\
& \leq c \sup _{0<s<t}\left(s^{\frac{1}{2}-\frac{n}{2 p_{1}}+\frac{n}{2 p}}\|\mathcal{F}(s)\|_{\dot{B}_{p_{1} \infty}^{\alpha}}\right) \int_{0}^{t}(t-s)^{-\frac{1}{2}-\frac{n}{2}\left(\frac{1}{p_{1}}-\frac{1}{p}\right)} s^{-\frac{1}{2}+\frac{n}{2 p_{1}}-\frac{n}{2 p}} d s \\
& =c \sup _{0<s<t}\left(s^{\frac{1}{2}-\frac{n}{2 p_{1}}+\frac{n}{2 p}}\|\mathcal{F}(s)\|_{\dot{B}_{p_{1} \infty}^{\alpha}}\right) .
\end{aligned}
$$

Hence, we obtain (2) of Lemma 3.3 for $0<\alpha<1$. 
From (C.8), (C.10), Lemma C.1 and (2.3), for $1<\alpha<2$ and $1<p_{1}<p$ with $1+\frac{n-1}{p}<\frac{n}{p_{1}}<$ $1+\frac{n}{p}$, we have

$$
\left\|\Gamma^{*} *(\mathbb{P} f)(t)\right\|_{\dot{B}_{p \infty}^{\alpha}} \leq \int_{0}^{t}(t-s)^{-\frac{1}{2}-\frac{n}{2}\left(\frac{1}{p_{1}}-\frac{1}{p}\right)}\|F(s)\|_{\dot{B}_{p_{1} \infty}^{\alpha}} d s
$$

As the same estimate of (C.11), we complete the proof of (2) of Lemma 3.3 .

\section{REFERENCES}

[1] M.F. de Almeida and L.C.F. Ferreira, On the Navier-Stokes equations in the half-space with initial and boundary rough data in Morrey spaces, J. Differential Equations, 254, no. 3, 1548-1570(2013).

[2] H. Amann, On the strong solvability of the Navier-Stokes equations, J. Math. Fluid Mech. 2, no. 1, 16-98 (2000).

[3] J. Bergh and J. Löfström, Interpolation Spaces. An Introduction, Grundlehren der Mathematischen Wissenschaften, No. 223. Springer-Verlag, Berlin-New York, 1976.

[4] M. Cannone, F. Planchon and M. Schonbek, Strong solutions to the incompressible Navier-Stokes equations in the half-space, Comm. Partial Differential Equations, 25, no. 5-6, 903-924(2000).

[5] D. Chae, Local existence and blow-up criterion for the Euler equations in the Besov spaces, Asymptotic Analysis, 38, 339-358 (2004).

[6] T. Chang and B.J. Jin, Solvability of the initial boundary value problem of the NavierStokes equations with rough data, Nonlinear Anal., 125, 498-517(2015).

[7] T. Chang and B. Jin, Initial and boundary values for $L_{\alpha}^{p}\left(L^{p}\right)$ solution of the Navier-Stokes equations in the halfspace, J. Math. Anal. Appl., 439, no. 1, 70-90(2016).

[8] T. Chang and B. Jin, Initial-boundary value problem of the Navier-Stokes equations in the half space with nonhomogeneous data, Ann. Univ. Ferrara Sez. VII Sci. Mat., 65, no. 1, 29-56(2019).

[9] T. Chang and B. Jin, Global in time solvability of the Navier-Stokes equations in the half-space, J. Differential equations, 267, no 7, 4293-4319(2019).

[10] T. Chang and K. Kang, Estimates of anisotropic Sobolev spaces with mixed norms for the Stokes system in a halfspace, Ann. Univ. Ferrara Sez. VII Sci. Mat. 64, no. 1, 47-82(2018).

[11] T. Chang and K. Kang, Solvability for Stokes system in Hölder spaces in bounded domains and its applications, J. Math. Fluid Mech., 20, no. 4, 18571888(2018).

[12] F. Crispo, P. Maremonti, On the $(x, t)$ asymptotic properties of solutions of the Navier-Stokes equations in the half space, Zap. Nauchn. Sem. S.-Peterburg. Otdel. Mat. Inst. Steklov. (POMI) 318 (2004), Kraev. Zadachi Mat. Fiz. i Smezh. Vopr. Teor. Funkts. 36 [35], 147-202, 311; reprinted in J. Math. Sci. (N.Y.) 136(2) (2006) 3735-3767.

[13] R. Danchin and P. Zhang, Inhomogeneous Navier-Stokes equations in the half-space, with only bounded density, J. Funct. Anal., 267, no. 7, 2371-2436 (2014).

[14] R. Farwig, Y. Giga and P.-Y. Hsu, Initial values for the Navier-Stokes equations in spaces with weights in time, Funkcialaj Ekvacioj, 59(2), 199-216(2016).

[15] R. Farwig, Y. Giga and P. Hsu, The Navier-Stokes equations with initial values in Besov spaces of type $B_{q \infty}^{-1+\frac{3}{q}}$, J. Korean Math. Soc. 54, no. 5, 1483-1504(2017).

[16] R. Farwig, H. Sohr and W. Varnhorn, On optimal initial value conditions for local strong solutions of the NavierStokes equations, Ann. Univ. Ferrara Sez. VII Sci. Mat., 55(1),89-110(2009).

[17] M. Giga, Y. Giga and H. Sohr, $L^{p}$ estimates for the Stokes system, Lect. Notes Math. 1540, 55-67(1993).

[18] Y. Giga and H. Sohr, Abstract $L^{p}$ estimates for the Cauchy problem with applications to the Navier-Stokes equations in exterior domains, J. Funct. Anal., 102, no. 1, 72-94(1991).

[19] H. Koch and V.A. Solonnikov, $L_{p}$-estimates of the first-order derivatives of solutions to the nonstationary Stokes problem. (English summary) Nonlinear problems in mathematical physics and related topics, I, Int. Math. Ser. (N. Y.), 1, Kluwer/Plenum, New York, 203-218( 2002).

[20] H. Koch and V. A. Solonnikov, $L_{p}$-Estimates for a solution to the nonstationary Stokes equations, J. Math. Sci, 106, No.3, 3042-3072(2001).

[21] H. Kozono, Global $L^{n}$-solution and its decay property for the Navier-Stokes equations in half-space $\mathbb{R}_{+}^{n}$, J. Differential Equations, 79, no. 1, 79-88(1989).

[22] H. Kozono and S. Shimizu, NavierStokes equations with external forces in time-weighted Besov spaces, Math. Nach, 291, 1781-1800( 2018). 
[23] H. Kozono and S. Shimizu, Strong solutions of the NavierStokes equations based on the maximal Lorentz regularity theorem in Besov spaces, Journal of Functional Analysis, 276, 896-931(2019).

[24] H. Kozono and M. Yamazaki, Local and global unique solvability of the Navier-Stokes exterior problem with Cauchy data in the space $L^{n, \infty}$. (English summary), Houston J. Math. 21, no. 4, 755-799(1995).

[25] P. Maremonti, Regular solutions to the Navier-Stokes equations with an initial data in L(3, $\infty)$, Ric. Mat. 66, no. 1 , 6597(2017).

[26] M. Ri, P. Zhang and Z. Zhang, Global well-posedness for Navier-Stokes equations with small initial value in $B_{n, \infty}^{0}(\Omega)$, J. Math. Fluid Mech., 18, no. 1, 103-131(2016).

[27] V. Solonnikov, Estimates for solutions of nonstationary Navier-Stokes equations, (Russian) Boundary value problems of mathematical physics and related questions in the theory of functions, 7. Zap. Naucn. Sem. Leningrad. Otdel. Mat. Inst. Steklov. (LoMI)38: p. 153-231 (1973).; translated in J. Soviet Math., 8, 467-529(1977).

[28] V.A. Solonnikov, Estimates of the solutions of the nonstationary Navier-Stokes system, Boundary value problems of mathematical physics and related questions in the theory of functions, 7. Zap. Naun. Sem. LOMI. 38, 153231(1973).

[29] V.A. Solonnikov, Estimates for solutions of the nonstationary Stokes problem in anisotropic Sobolev spaces and estimates for the resolvent of the Stokes operator, (Russian) Uspekhi Mat. Nauk 58 (2003), no. 2(350), 123-156; translation in Russian Math. Surveys 58 (2003), no. 2, 331-365.

[30] E.M. Stein, Singular Integrals and Differentiability Properties of Functions, Princeton Mathematical Series, No. 30 Princeton University Press, Princeton, N.J. 1970.

[31] H. Triebel, Interpolation Theory, Function Spaces, Differential Operators, Second edition. Johann Ambrosius Barth, Heidelberg, 1995.

Department of Mathematics, Yonsei University, Seoul, 136-701, South Korea

E-mail address: chang7357@yonsei.ac.kr

Department of Mathematics, Mokpo National University, Muan-Gun 534-729, South Korea

E-mail address: bumjajin@mokpo.ac.kr 